\title{
Maturation of corticospinal tracts assessed by electromagnetic stimulation of the motor cortex
}

\author{
T H H G KOH AND J A EYRE \\ Department of Child Health, University of Newcastle upon Tyne
}

SUMmARY The motor cortex can be excited in adults using electromagnetic stimulation, and the latency to the evoked muscle action potential allows an assessment of the integrity of corticospinal tracts. We applied this technique in children to describe the maturation of corticospinal tracts. The latency from cortical stimulation to the onset of the evoked muscle action potentials and the subject's height were recorded. The subject's height was divided by the latency to the onset of the evoked muscle action potential to provide an index of the conduction velocity within descending motor pathways $\left(\mathrm{V}_{\mathrm{I}}\right)$. It is possible to evoke muscle action potentials after electromagnetic stimulation of the motor cortex in children including preterm babies and there is a stepwise increase in the sensitivity to stimulation between 8 and 11 years of age. In addition there is a progressive increase in $V_{I}$ with age; adult values are attained at about 11 years.

The successful application of this technique in children suggests that electromagnetic stimulation of the motor cortex has the potential to allow detection of abnormality in motor pathways in newborn babies and young children.

In young children it is difficult to assess the integrity of central motor pathways. Clinical examination is unreliable particularly in the very young child or in the newborn infant. ${ }^{1}$ The recent development of the technique of electromagnetic stimulation of the motor cortex provides, for the first time, the opportunity to assess objectively the integrity of corticospinal tracts in children. ${ }^{2}$ This method uses a flat circular stimulating coil through which a brief current is passed. ${ }^{2}$ The pulsed magnetic field so generated induces electrical currents in the brain, which are assumed to excite the superficial layers of the cortex. When the coil is held over the motor cortex, activity is evoked in the corticospinal tracts and results in muscle action potentials, measureable by surface electromyogram electrodes. ${ }^{2}{ }^{3}$ The method is simple, painless, and quick and is therefore particularly suitable for use in children. Before this technique can be used to investigate abnormalities in motor pathway function in children, however, the normal developmental changes in the conduction velocity within the motor pathways need to be defined.

The aim of this study was, therefore, to define the change with age in the conduction velocity in descending motor pathways after electromagnetic stimulation of the motor cortex in healthy subjects.

\section{Subjects and methods}

This study obtained the approval of the Newcastle Health Authority ethical committee after the evaluation of the safety of the technique in an animal model ${ }^{4}$ We studied 142 subjects who ranged in age from 33 weeks' gestation to 50 years; they were healthy with no history or clinical signs of a neurological disorder. Informed consent was obtained from subjects aged greater than 16 years and from the parents of children aged less than 16 years.

To record muscle action potentials, skin mounted electromyogram electrodes were placed over the right abductor digiti minimi in all subjects older than 6 months of age; in those aged less than 6 months the muscle action potential was recorded from the right biceps brachii. The electromyogram was amplified by $10^{4}$ by a Nicolet Physiological amplifier (NIC-1001A) and filtered using a band pass of $5 \mathrm{~Hz}$ to $1.5 \mathrm{kHz}$. The amplifier was interfaced to a microcomputer for storage of the wave form and for subsequent analysis.

To stimulate the motor cortex, a high current low voltage magnetic stimulator ${ }^{5}$ was used to generate a pulsed magnetic field of up to 1.9 tesla (T), with a rise time of $100 \mu \mathrm{s}$ and total duration of $300 \mu \mathrm{s}$. The 
characteristics of the magnetic field generated are shown in fig 1 . The stimulating coil was held tangential to the scalp and positioned to obtain the maximum muscle action potential in each subject.

The study protocol was to attempt to evoke muscle action potentials after electromagnetic stimulation of the cortex in relaxed abductor digiti minimi (or biceps brachii in those less than 6 months old). Merton and his colleagues have shown that the threshold for evoking muscle action potentials can be lowered if there is isometric contraction in the relevant muscle. ${ }^{6}$ Therefore, if it was not possible to evoke a muscle action potential in relaxed muscle isometric contraction of the abductor digiti minimi in subjects older than 6 months was achieved by placing a weight in the palm of the outstretched and supinated right hand; in those aged less than 6 months reflexive isometric contraction in biceps brachii was induced by eliciting a traction reflex. ${ }^{7}$

The latency from cortical stimulation to the onset of the evoked muscle action potential was determined and the subject's standing height, or crown to heel length in those less than 1 year old, were recorded.

\section{Results}

Representative recordings of evoked muscle action

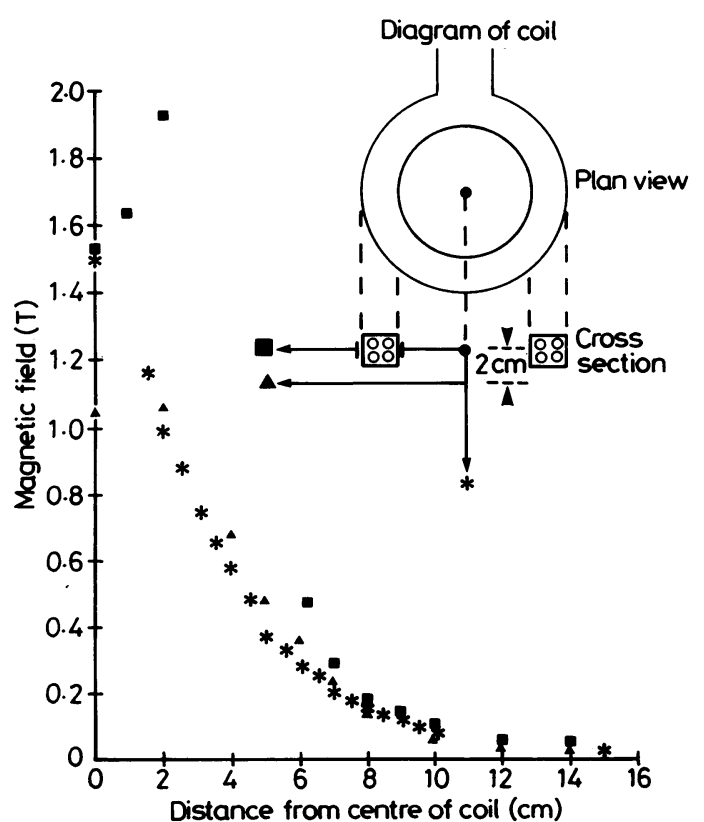

Fig 1 Intensity of the magnetic field generated by the stimulating coil in three planes in relation to the centre of the coil. potentials after electromagnetic stimulation of the motor cortex in subjects aged 5 days, 5 years, 10 years, 16 years, and 23 years are shown in fig 2 .

Muscle action potentials were evoked in all 142 subjects; the facilitation of an isometric contraction was required in 71 . The incidence of obtaining a evoked muscle action potential from relaxed muscle in relation to the subjects' ages is shown in fig 3 . All the subjects aged less than 8 years required the facilitation of an isometric contraction. There was a stepwise increase in the incidence of obtaining muscle action potentials in relaxed muscle between the ages of 8 and 11 years but it was not until 20 years of age that muscle action potentials could be consistently obtained in relaxed muscle.

The mean (SD) latency to the evoked muscle action potential in subjects older than 20 years was 21 (1) ms. These data are consistent with those obtained in healthy adult subjects by previous

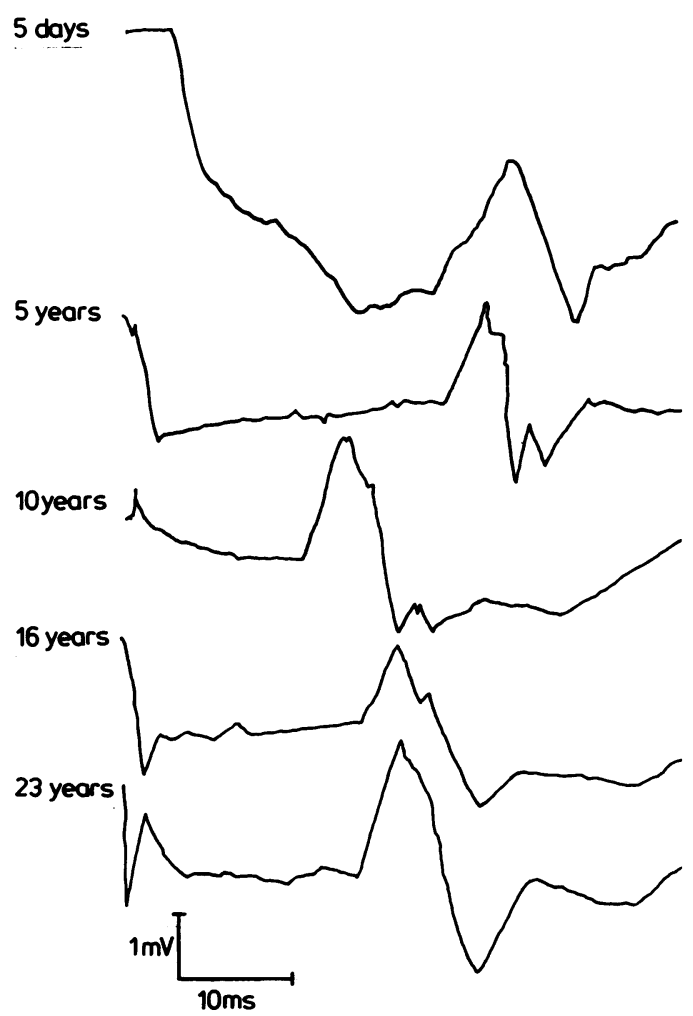

Fig 2 Representative recordings of muscle action potentials evoked after electromagnetic stimulation of the motor cortex in subjects of differing ages. The muscle action potential was recorded from abductor digiti minimii in all except the subject of 5 days of age where it was recorded from biceps brachii. 


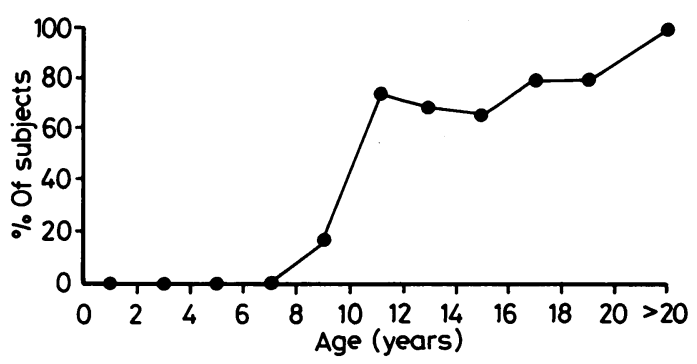

Fig 3 Incidence of obtaining an evoked muscle action potential in the relaxed muscle in relation to age. workers. ${ }^{2}$ There was a very wide variability in the latency to evoked muscle action potential in children. There was, however, a reduction in this latency with age from a mean (SD) of $31(1 \cdot 1) \mathrm{ms}$ in term babies (fig 4).

The latency to the onset of a muscle action potential is dependent on the conduction velocity within the descending motor pathways and the distance travelled. It is not possible to measure accurately the distance from the motor cortex to a peripheral muscle in living subjects. To provide an estimate of this distance the subjects height or crown to heel length was used. An index of the

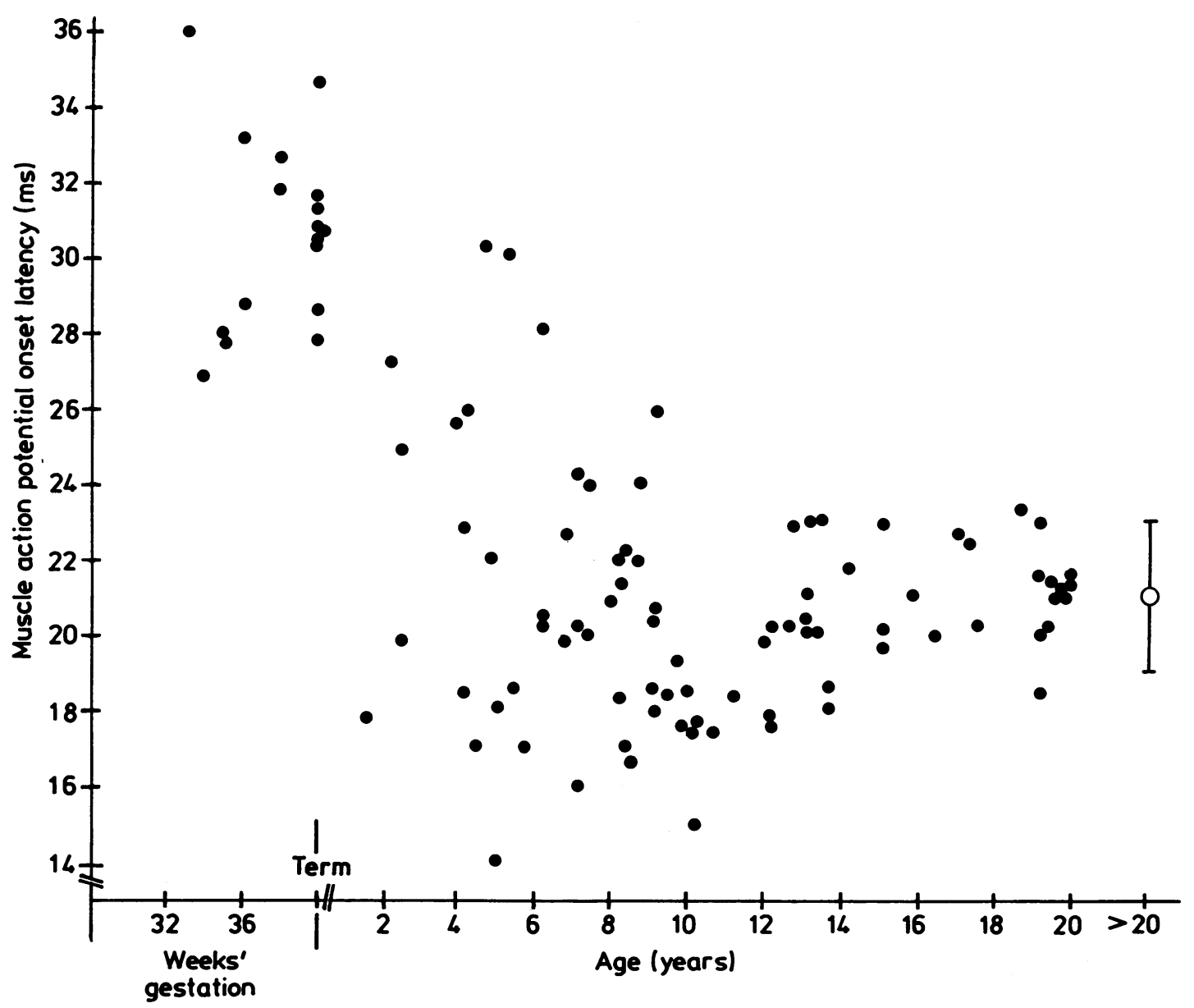

Fig 4 Latency to the onset of an evoked muscle action potential in abductor digiti minimi in relation to age. The latency for the subjects less than 6 months is derived from the latency to biceps brachii plus the estimated conduction time from biceps brachii to abductor digiti minimi. The mean (2SD) for the 30 subjects $>20$ years old is indicated on the right. 


\section{Koh and Eyre}

conduction velocity in corticospinal tracts $\left(\mathrm{V}_{\mathrm{I}}\right)$ was calculated by dividing the height or crown to heel length by the latency to the onset of the evoked muscle action potential. These data are shown in relation to the subject's age in years in fig 5 . This shows a progressive increase in $V_{I}$ with age from $10.5 \mathrm{~m} / \mathrm{s}$ at 33 weeks' gestation until adult values $(79(10) \mathrm{m} / \mathrm{s})$ are reached by the ages of 11 years.

\section{Discussion}

This study has shown for the first time that it is possible to evoke activity in descending motor pathways after electromagnetic stimulation of the motor cortex in premature and term newborn babies, infants, and older children.

Currently it is uncertain which neural elements in the motor cortex are excited by the stimulus. ${ }^{8}$ There is evidence, however, to suggest that the electromagnetic stimulus excites interneurons in the motor cortex, resulting in indirect rather than direct activation of the pyramidal neurons. ${ }^{9}$

An unexpected finding in our present study was the need for an isometric contraction in order to evoke a muscle action potential after electromagnetic stimulation of the motor cortex in all children aged less than 8 years. This need for facilitation suggests that the cortical interneurons or pyramidal neurons have a higher threshold for activation in young children than in adults or that the alpha motor neurons are less readily excited by the activity evoked in the descending motor pathways after electromagnetic stimulation. The sudden increase in the incidence of obtaining a muscle action potential in relaxed muscle between the ages of 8 and 11 years further suggests that during this period there is a stepwise decrease in the threshold for the activation of cortical neurons or alpha motor neurons or both.

Bruce and Tatton, using microelectrical stimulation of the cortex in the kitten, have described a

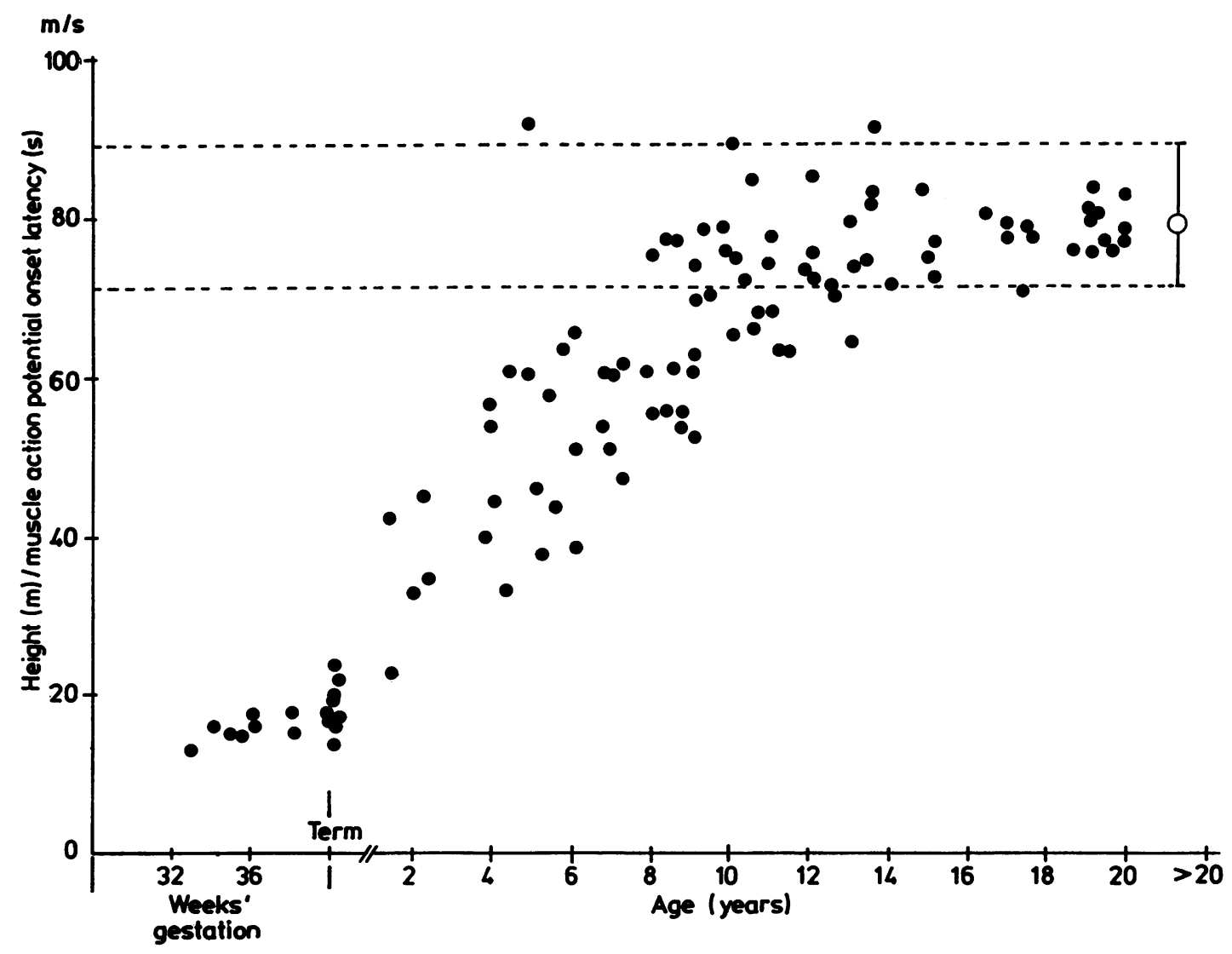

Fig 5 Correlation of $V_{I}$ (height or crown to heel length/latency to the onset of the evoked muscle action potential) with age. 
stepwise change with age in the sensitivity of the neurons in the cortical motor area 4 to electrical stimulation..$^{10}$ These data suggest that at least part of the maturational change in threshold for evoking muscle action potentials observed may be cortical in origin.

At present most authors only consider the latency of the muscle action potential for determining normal and abnormal responses. ${ }^{2} 38$ Our data show that it is also important to consider the threshold for evoking muscle action potential in defining both normal and abnormal responses.

In man the latency from cortical stimulation to evoked activity in the cervical spine is about $6 \mathrm{~ms} .^{8}$ This short latency suggests that the large myelinated axons of the corticospinal tract are concerned in the conduction of descending activity after electromagnetic stimulation of the motor cortex. This conclusion is supported by studies in primates in which short latency neural activity has been recorded in the lateral corticospinal tract after electromagnetic stimulation of the motor cortex. ${ }^{9}$

We have shown a progressive increase in the index of conduction velocity $\left(V_{I}\right)$ within the descending motor pathways up until the age of 11 when adult values are achieved. It has been established that the conduction velocities in peripheral motor nerves reach adult values by 3 years. ${ }^{11}$ Histochemical studies of postmortem material have shown that myelination of corticospinal tracts is complete by 3 years of age. ${ }^{12}$ Our data suggest that there is an as yet uncharacterised process of maturation that leads to an apparent increase in the conduction velocity within the corticospinal tract beyond the completion of myelination. The decrease with age in the latency to the evoked muscle action potential may be due, in part, to the development and maturation of interneuronal synaptic connections both at the cortex and the spinal cord. This conclusion is supported by our finding of the change with age in the threshold for evoking muscle action potentials.

The normal development of motor behaviours has been studied in detail ${ }^{13}$; these studies have shown continued acquisition of fine motor skills up until the age of $8-10$ years. ${ }^{14}$ Our findings provide corresponding neurophysiological evidence of continuing maturation of central motor pathways up until the ages of 11 years.

Desmedt and his colleagues have studied the maturation of the conduction velocities within central somatosensory pathways. ${ }^{15}$ These studies have shown a similar pattern of maturation with adult values being reached by 8 years of age.

Finally it has been shown in adults that prolongation of the latency to evoked muscle action potential after electromagnetic stimulation of the cortex is a sensitive indicator of abnormality in descending motor pathways. ${ }^{23}$ The electromagnetic stimulation of the cortex was well tolerated by all the subjects in our study including the very young children and the newborn infants. The ease in the use of this technique in children suggests that it may provide an exciting opportunity for the objective assessment of motor pathway dysfunction in young children and in particular for the early detection of cerebral palsy in the newborn baby.

A study is presently being conducted to assess the sensitivity of this technique for the detection of upper motor neuron lesions in newborn infants and young children.

We would like to thank the following for their support and help: the subjects and their parents, Professor S Miller, Professor A Aynsley-Green, Dr D Milligan, Mr P Byrne, Mr P Oliver, Mr T Essex, and Mrs B Kenyon. Financial support is acknowledged from the Mason Medical Foundation and The Newcastle Health Authority. THHG Koh is a Medical Research Council Training Fellow.

Dr J Eyre is a Wellcome Senior Fellow in Clinical Science.

\section{References}

${ }^{1}$ Illingworth RS. The diagnosis of cerebral palsy in the first year of life. Dev Med Child Neurol 1966;8:178-94.

2 Barker AT, Freestone IL, Jalinous R, Jarratt JA. Magnetic stimulation of the human brain and peripheral nervous system: an introduction and the results of an initial clinical evaluation. Neurosurgery 1987;20:100-9.

${ }^{3}$ Hess CW, Mills KR, Murray NMF. Measurement of central motor conduction in multiple sclerosis by magnetic brain stimulation. Lancet 1986;ii:355-8.

4 Eyre JA, Flecknall PA, Kenyon BR, Koh THHG, Miller S. Effects of electromagnetic stimulation of the brain on cortical activity, cortical blood flow, blood pressure and heart rate in the cat. J Physiol (Lond) 1988;396:154P.

5 Byrne PO, Eyre JA, Kenyon BR, Koh THHG, Miller S, Oliver PD. High current discharge circuit for electromagnetic stimulation of the brain in man. J Physiol (Lond) 1987;391:3P.

6 Merton PA, Hill DK, Morton HB, Marsden HB. Scope of a technique for electrical stimulation of human brain, spinal cord and muscle. Lancet 1982;ii:597-600.

${ }^{7}$ Prechtl H. The neurological examination of the full term newborn infant. London: Spastics International Medical Publications, 1977:46.

${ }^{8}$ Mills KR, Murray NMF, Hess CW. Magnetic and electrical transcranial brain stimulation: physiological mechanisms and clinical applications. Neurosurgery 1987;20:164-8.

9 Amassian VE, Quirk GJ, Stewart M. Magnetic coil versus electrical stimulation of monkey motor cortex. J Physiol (Lond) 1987;394:119P.

10 Bruce IC, Tatton WG. Sequential output-input maturation of kitten motor cortex. Exp Brain Res 1980;39:411-9.

11 Thomas JE, Lambert EH. Ulnar nerve conduction velocity and H-reflex in infants and children. J Appl Physiol 1960;15:1-9.

12 Yakovlev PI, Lecours A-R. The myelogenetic cycles of regional maturation of the brain. In: Minkowski A, ed. Regional development of the brain in early life. Oxford: Blackwell Scientific Publications, 1967:3-70.

13 Illingworth RS. The development of the infant and young child, normal and abnormal. Edinburgh: Livingstone, 1966. 


\section{Koh and Eyre}

14 Denckla MB. Development of motor co-ordination in normal children. Dev Med Child Neurol 1974;16:729-41.

15 Desmedt JE, Brunko E, Debecker J. Maturation of the somatosensory evoked potentials in normal infants and children, with special reference to the early $\mathrm{N}_{\mathrm{I}}$ component. Electroencephalogr Clin Neurophysiol 1976;40:43-58.

Correspondence to Dr THHG Koh, Department of Child Health, University of Newcastle upon Tyne, Newcastle upon Tyne NE2 4HH.

Accepted 27 June 1988 